\title{
Os efeitos dos aparelhos funcionais sobre a dimensão transversal da maxila e mandíbula
}

\author{
Adriano Marotta Araújo*, Peter H. Buschang**
}

\begin{abstract}
Resumo
Enquanto os efeitos dos aparelhos ortopédicos funcionais sobre as dimensões ântero-posterior e vertical vêm sendo amplamente estudados, o efeito sobre a dimensão transversal é um campo limitado e pouco explorado. A presença de escudos vestibulares, a pressão muscular, tração periostal e o reposicionamento lingual são alguns dos tópicos que podem influenciar o crescimento transversal da maxila e mandíbula. Vários estudos mostram uma alteração transversal dos arcos dentários, como resposta da terapia com o aparelho funcional. Baseado em uma revisão sistemática da literatura esse trabalho teve como objetivo: 1) avaliar os mecanismo de ação dos aparelhos e a influência sobre a dimensão transversal e 2) resumir os efeitos clínicos de diferentes aparelhos funcionais sobre a dimensão transversal.
\end{abstract}

Palavras-chave: Aparelho funcional. Expansão maxilar. Expansão mandibular. Má oclusão Classe II.

\section{INTRODUÇÃO}

Devido ao grande número de estudos realizados com os aparelhos funcionais avaliando os efeitos dos aparelhos nos sentidos sagital e vertical, hoje sabemos que essa terapia é capaz de alterar e até redirecionar o crescimento da maxila e mandíbula em algum grau. Contudo, quando considerada a dimensão transversal, poucos foram os trabalhos elaborados e conseqüentemente alguns tópicos ainda não foram totalmente esclarecidos.

Alguns autores defendem a teoria no qual os "escudos vestibulares", presentes nos aparelhos, são capazes de alterar a dimensão transversal dos arcos dentários 6,7,9,12,19. De acordo com Frankel ${ }^{6}$, "o regulador funcional em sua constituição apre- senta escudos vestibulares que promovem uma expansão e remodelação dentoalveolar devido a uma eliminação da pressão dos tecidos moles adjacentes e à aplicação de uma tensão periostal sobre o tecido ósseo. Isto permite um desenvolvimento normal dos arcos dentários com uma erupção dos dentes permanentes num sentido mais vestibular. A extensão apical dos escudos vestibulares capazes de gerar uma tensão nas fibras musculares e periósteo estimula a aposição óssea na região, assim associado a correção ântero-posterior ocorre uma expansão do arco dentário superior e inferior".

Graber e Vanarsdall ${ }^{13}$ citaram que a ação dos escudos vestibulares do aparelho bionator

\footnotetext{
* Doutor em Ortodontia - Faculdade de Odontologia de Araraquara - UNESP. Fellowship no Departamento de Ortodontia da Baylor College of Dentistry - Dallas, Tx. Prof. Titular na disciplina de Ortodontia no curso de Graduação - UNISA - SP.

** Ph.D em Antropologia Física pela Universidade do Texas e Diretor Científico do Departamento de Ortodontia da Baylor College of Dentistry - Dallas, Tx.
} 
apresenta dois principais objetivos: 1) manter afastado o tecido mole da bochecha que normalmente é trazido para dentro do espaço interoclusal e 2) mover a superfície da cápsula oro-bucal lateralmente, aumentando o espaço bucal em virtude do posicionamento para frente da mandíbula, enquanto os arcos vestibulares a mantêm afastada da mucosa alveolar. Outros autores como Andresen e Balters (apud GRABER, VANARSDALL ${ }^{13}$, 1994) confirmam essa teoria e acreditam na eliminação da pressão dos músculos da bochecha e uma erupção mais vestibular dos dentes permanentes como responsáveis pela expansão dos arcos dentários. Mecanismo semelhante ocorre com a placa lábio ativa, a armação lateral do aparelho remove a pressão da musculatura da bochecha sobre os dentes posteriores permitindo a ação muscular da língua gerando um aumento das dimensões transversais no arco dentário ${ }^{4,18}$. Porém, em contradição, Trenouth ${ }^{26}$ não acredita nessa possibilidade de tratamento e recomenda a expansão mecânica numa fase anterior ao uso do aparelho funcional, pois acredita ele que desta maneira a quantidade de expansão pode ser precisamente calculada.

Podemos observar na literatura certa variabilidade de opiniões. Por exemplo, pesquisadores questionam se caso a expansão realmente ocorre com o uso de aparelhos funcionais, onde ela realmente se apresenta? A expansão acontece somente em nível alveolar ou também se alteram as bases ósseas? A deposição óssea realmente acontece após a tração periostal com os aparelhos funcionais? Qual é o real mecanismo de ação dos escudos vestibulares? A terapia é capaz de alterar o padrão de erupção dentária?

O propósito desse trabalho foi realizar uma discussão, com base na literatura, dos efeitos transversais na maxila e na mandíbula com o uso de aparelhos ortopédicos funcionais e avaliar a oportunidade real de uso clínico dessa modalidade de tratamento. $\mathrm{O}$ mesmo foi dividido em duas partes, inicialmente avaliamos a influência da musculatura peribucal no desenvolvimento transversal em estudos experimentais realizados com animais, focalizando a ação dos escudos vestibulares, a ação muscular da língua e aposição óssea como conseqüência da tração periostal. E em uma segunda parte discutimos a ação dos aparelhos funcionais em trabalhos clínicos realizados em seres humanos.

\section{INFLUÊNCIA DA MUSCULATURA PERIBU- CAL SOBRE A DIMENSÃO TRANSVERSAL DA MAXILA E MANDÍBULA EM ESTUDOS EXPERIMENTAIS COM ANIMAIS}

Alguns autores afirmam que a tração periostal provocada pelo uso do aparelho é capaz de expandir os arcos dentários e, ainda, que a expansão obtida não se restringe somente aos arcos dentários, mas também às bases ósseas da maxila e mandíbula $a^{3,6,7,17,19}$. Outros afirmam que a etiologia para expansão não é a tração periostal e sim a eliminação da pressão vestibular da musculatura associada à pressão da língua ${ }^{5,6,8,9}$. A avaliação de uma seqüência de estudos experimentais nos auxiliou a compreender até que ponto a musculatura é capaz de influenciar a dimensão transversal e a forma do arco como um todo.

Ghafari e Shanfeld ${ }^{10}$, em um estudo realizado com ratos, concluíram que a forma do arco dentário está diretamente relacionada com a função da musculatura. Doze ratos da raça "sprague dawley" foram igualmente divididos em dois grupos, grupo experimental e controle. Nos animais do grupo experimental foi instalado um aparelho com escudos vestibulares com o objetivo de manter a musculatura afastada $1,5 \mathrm{~mm}$ do arco dentário maxilar. Os animais foram sacrificados em diferentes períodos - 1, 5, 15, 30, 40 dias após a instalação do aparelho. Após 5 dias foi encontrado aumento transversal do arco dentário para o grupo experimental e após 15 dias essa diferença foi estatisticamente significante; com o tempo a expansão apresentou valores menores. 
Desta maneira, a expansão obtida foi resultado da remoção da pressão da musculatura vestibular sobre o arco dentário maxilar.

Três anos mais tarde o mesmo autor investigou as alterações ocorridas na sutura palatina após a remoção da pressão muscular ${ }^{9}$. $\mathrm{O}$ estudo histológico pareceu corresponder com macromorfológico realizado anteriormente, onde foi observado um aumento gradativo transversal do arco. Os resultados levaram o autor a concluir que a cartilagem secundária da sutura palatina em ratos é de natureza adaptativa. Essa adaptação acontece quando a sutura é submetida ao estresse mecânico e/ou decorrente do potencial de crescimento.

Sotiriadou e Johnston ${ }^{25}$ avaliaram a efetividade dos escudos vestibulares testando duas diferentes hipóteses: 1) A expansão do arco dentário é resultado do desequilíbrio funcional com a língua exercendo maior pressão sobre os dentes ou 2) A tração periostal sobre o tecido ósseo é responsável pela expansão do arco dentário. Quarenta e quatro ratos albinos foram divididos em 4 grupos. Para eliminar o efeito da língua sobre os dentes em metade da amostra foi cortada a coroa dos molares, assim 4 grupos foram formados: Grupo "SM" - foram adaptados os escudos vestibulares e os molares permaneceram intactos; Grupo "Sm" - presença dos escudos vestibulares e ausência das coroas dos molares; Grupo "sM" - ausência dos escudos vestibulares e molares intactos e, por fim, Grupo "sm" - com ausência dos escudos e das coroas dentárias. Os resultados revelaram existir uma expansão basal e dentária significante para os quatro grupos, isso demonstrou a influência do fator intrínseco sobre a expansão. Alta correlação foi detectada entre os escudos vestibulares e molares intactos, ou seja, os escudos vestibulares provocaram uma sobre expansão somente quando os ratos estavam com os molares intactos. Os autores concluíram que provavelmente a expansão foi resultado da pressão da língua sobre os dentes e não um resultado da tensão periostal.

O desenvolvimento transversal do arco dentário, a remodelação óssea da superfície lateral do processo alveolar e a sutura palatina foram avaliadas após tracionamento da musculatura com o uso de escudos vestibulares num estudo biométrico e histológico realizado em coelhos ${ }^{16}$. Dez coelhos da raça "new zealand", machos e com 12 semanas de idade foram utilizados. Os animais foram divididos em 2 grupos, controle e experimental. No grupo experimental, um aparelho confeccionado com escudos vestibulares foi instalado impedindo a ação dos músculos sobre o arco dentário. Os resultados da análise biométrica mostraram expansão do arco dentário nos dois grupos, porém significativamente maior para o grupo experimental. Microscopicamente também foi observado crescimento significante da sutura palatina para o grupo experimental. Nenhuma diferença significante foi encontrada no processo alveolar da maxila entre os dois grupos. Os resultados apresentados permitiram aos autores concluirem que os escudos vestibulares causaram uma expansão transversal no arco dentário maxilar com aumento do crescimento da sutura palatina. Não foi encontrada deposição óssea na região dentoalveolar, tornando discutível a teoria que a tração periostal causa uma expansão no arco dentário.

Nos parece coerente afirmar que a presença dos escudos vestibulares é capaz de alterar a dimensão transversal dos arcos dentários. A origem dessa expansão foi discutida por vários autores e possivelmente essa expansão foi resultado da eliminação das forcas musculares vestibulares com ação conjunta da pressão lingual associado ao fator intrínseco natural. Nos animais o crescimento sutural respondeu ao estimulo proveniente dos aparelhos, sendo este adaptativo e relacionado com o potencial de crescimento individual. A tensão periostal por si só pareceu não ser capaz de expandir os arcos dentários. 
INFLUÊNCIA DOS APARELHOS FUNCIONAIS SOBRE O DESENVOLVIMENTO TRANSVERSAL DA MAXILA E MANDÍBULA

Os estudos realizados em animais são de grande importância na compreensão do mecanismo de ação e avaliação dos efeitos de uma determinada terapia, medicamento ou aparelho. Nesse
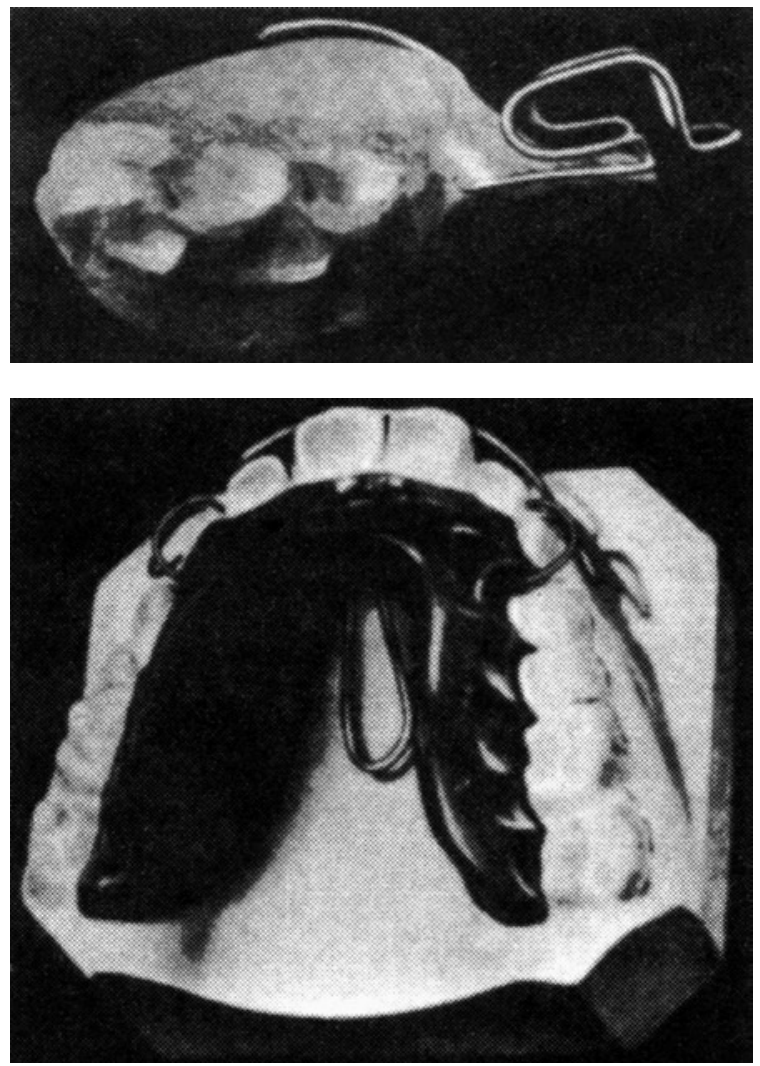

FIGURA 1 - Ativador Andresen-Häupl (GRABER, 1994).

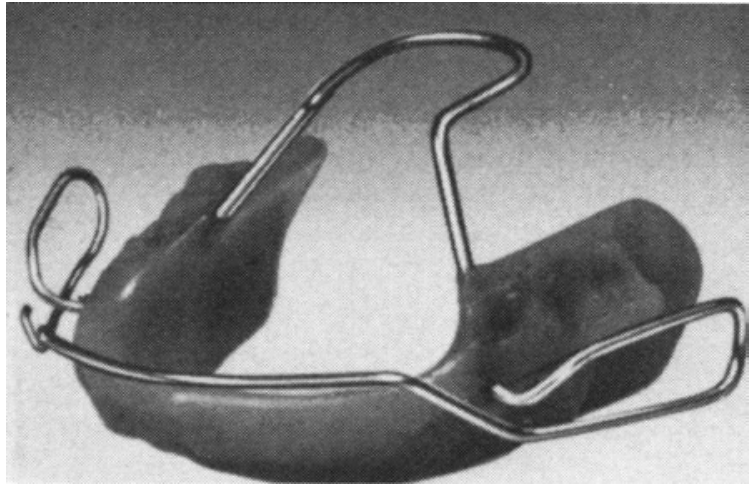

FIGURA 2 - Bionator de Balters (GRABER, 1994). caso em questão, onde estamos avaliando a ação de forcas ambientais sobre o crescimento transversal, os experimentos com animais nos auxiliou na identificação dos limites e áreas de atuação dos escudos vestibulares, porém os resultados obtidos não podem ser totalmente generalizados para os seres humanos. Algumas peculiaridades devem ser consideradas, tais como crescimento craniofacial, desenvolvimento da oclusão, tempo de tratamento, entre outras.

A seguir focalizamos na comparação de diferentes trabalhos clínicos realizados em seres humanos. Para facilitar a comparação, os resultados foram agrupados e resumidos na tabela 1. As figuras 1, 2 e 3 apresentam os aparelhos Andresen, bionator de Balters e o regulador funcional de Frankel respectivamente. Uma série de questões foi elaborada com o intuito de facilitar a discussão.

Os aparelhos funcionais são capazes de expandir os arcos dentários? Sim, os aparelhos funcionais são capazes de alterar clinicamente a dimensão transversal dos arcos dentários. A tabela 1 apresenta a anualização de grande parte dos trabalhos clínicos realizados com diferentes aparelhos funcionais. Após um tratamento em média de 12 meses foi observada uma expansão de 0,9 $\mathrm{mm}$ e $1,5 \mathrm{~mm}$ para as distâncias intercaninos e intermolares na maxila. A distância intercaninos inferiores quase não se alterou e a distância intermolares apresentou uma expansão de $0,7 \mathrm{~mm}$; todos os valores foram superiores quando compa-

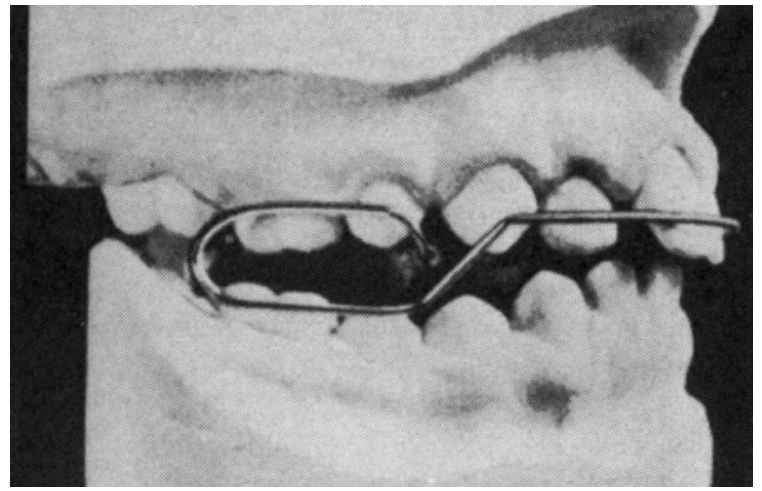


Tabela 1 - Resumo dos resultados dos trabalhos clínicos anualizados (mm/ano) com aparelhos funcionais e comparação com amostra controle (MOYERS $\left.{ }^{20}, 1976\right)$. ( $\mathrm{N}=$ número da amostra, Tx=tratamento, 3-3=distância intercaninos, 6-6=distância intermolares).

\begin{tabular}{|c|c|c|c|c|c|c|c|}
\hline \multicolumn{8}{|c|}{ Alterações transversais com aparelhos funcionais } \\
\hline \multirow{2}{*}{ Referências } & \multirow{2}{*}{ Aparelho } & \multirow{2}{*}{$\mathbf{N}$} & \multirow{2}{*}{ Idade } & \multicolumn{2}{|c|}{ Maxila } & \multicolumn{2}{|c|}{ Mandíbula } \\
\hline & & & & 3-3 & 6-6 & 3-3 & 6-6 \\
\hline Frankel (1971) & Frankel & 400 & - & - & 1,2 & - & - \\
\hline Mc Dougall et al. (1982) & Frankel & 60 & 9,0 & 1,0 & 1,8 & 0,6 & 1,1 \\
\hline & Frankel & 60 & 9,0 & 0,6 & 1,8 & 0,3 & 0,8 \\
\hline Owen (1983) & Frankel & 50 & 9,6 & - & 2,3 & - & 1,5 \\
\hline McWade et al.( 1987) & Frankel & 24 & 10,0 & 1,5 & 2,3 & 0,6 & 0,8 \\
\hline Hamilton et al. (1987) & Frankel & 25 & 9,9 & 1,0 & 1,1 & 0,8 & 0,5 \\
\hline Hime \& Owen (1990) & Frankel & 11 & 9,9 & - & - & 0,7 & 0,6 \\
\hline Gibbs \& Hunt (1992) & Frankel & 23 & $11-12$ & 1,1 & 1,4 & 0,1 & 0,5 \\
\hline Ghafari et al. (1998) & Frankel & 28 & $7-13$ & 0,9 & 1,4 & 0,2 & 0,9 \\
\hline Média & & & 9,8 & 1,0 & 1,6 & 0,5 & 0,8 \\
\hline Controle & & & 10 & 0,4 & 0,6 & 0,0 & 0,4 \\
\hline Pancherz (1976) & Andresen & 112 & 11,1 & 0,8 & 0,7 & $-0,2$ & 0,2 \\
\hline Reey \& Eastwood (1978) & Andresen & 31 & 11,0 & 0,9 & 1,9 & $-0,5$ & 0,9 \\
\hline Vargervik (1979) & Andresen & 55 & 9,6 & - & 1,3 & - & 0,4 \\
\hline Gibbs \& Hunt (1992) & Andresen & 27 & $11-12$ & 0,6 & 1,2 & $-0,2$ & 0,3 \\
\hline Média & & & 10,8 & 0,8 & 1,3 & $-0,3$ & 0,5 \\
\hline Controle & & & 11 & 0,4 & 0,5 & $-0,2$ & 0,3 \\
\hline Gibbs \& Hunt (1992) & Bionator & 19 & $11-12$ & 0,4 & 1,2 & $-0,1$ & 0,4 \\
\hline Almeida et al. (1998) & Bionator & 31 & 10,3 & 0,9 & 1,1 & 0,1 & 0,4 \\
\hline Dominguez et al. (2001) & Bionator & 31 & 10,7 & 0,5 & 1,1 & 0,2 & 0,9 \\
\hline Média & & & 10,8 & 0,6 & 1,1 & 0,1 & 0,5 \\
\hline Controle & & & 11 & 0,4 & 0,5 & $-0,2$ & 0,3 \\
\hline Controle & & & 10 & 0,4 & 0,6 & 0,0 & 0,4 \\
\hline Média Total & & & 10,3 & 0,9 & 1,5 & 0,2 & 0,7 \\
\hline
\end{tabular}
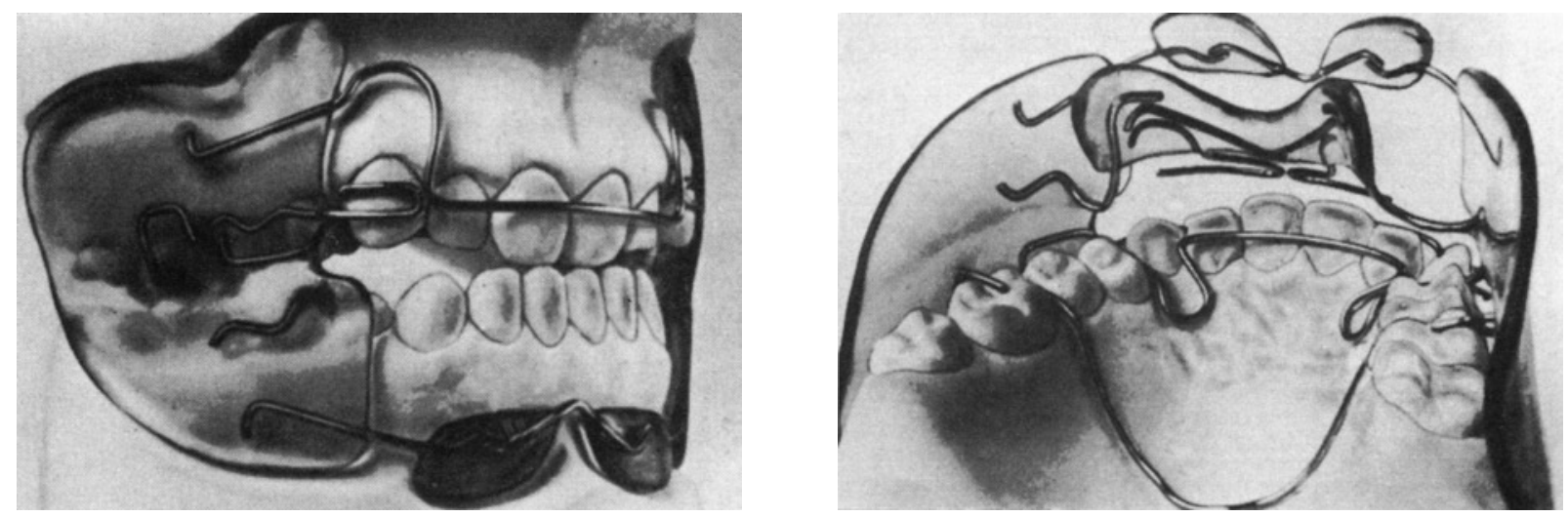

FIGURA 3 - Regulador funcional de Fränkel (GRABER, 1994). 


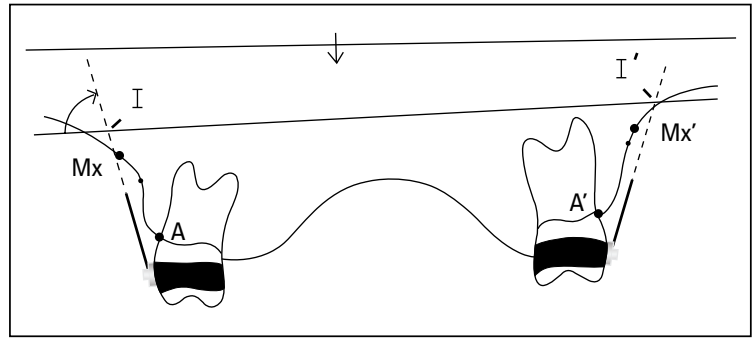

FIGURA 4 - Esquema ilustrando as mensurações transversais $(\mathrm{mm})$ da maxila (BRIEDEN et al., 1984). I-I': distância entre implantes metálicos posteriores / Mx-Mx': distância entre o ponto mais profundo da concavidade dentoalveolar da maxila do lado direito ao mesmo ponto do lado oposto / A-A': ponto na junção osso alveolar/dente do lado direito as mesmo ponto do lado oposto, conhecido como base apical.

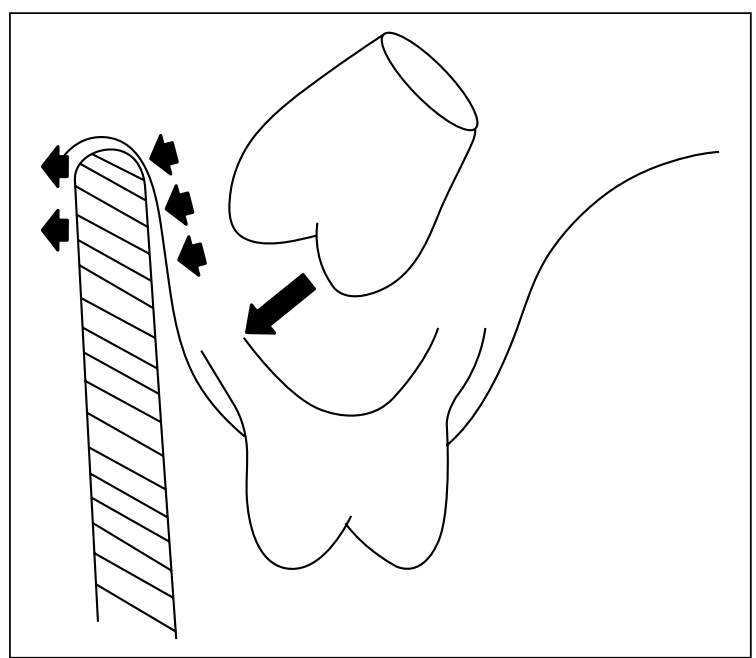

FIGURA 5 - Esquema representando a ação do escudo vestibular criando uma tensão no sentido lateral na mucosa peribucal na região dos molares superiores. Note que a tensão influencia a direção de erupção do dente permanente no sentido vestibular (setas menores) e pouca influencia o dente erupcionado (OWEN, 1983).

rados a uma amostra controle ${ }^{20}$.

Porque a maxila apresentou uma maior expansão quando comparada à expansão encontrada na mandíbula? Algumas explicações foram consideradas $^{15}:$ 1) a terapia com o ativador causa uma alteração postural e muscular na língua e na bochecha, com a língua ocupando uma posição mais elevada e anteriorizada, provocando uma redução na tensão dos músculos da bochecha sobre a região posterior do arco dentário maxilar e conseqüentemente permitindo uma maior expansão na região ${ }^{21}$, 2) a distância entre os escudos vestibulares e o

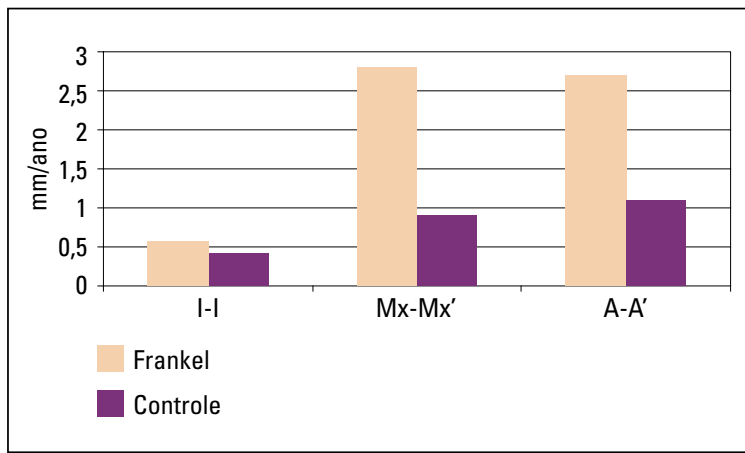

GRÁFICO 1 - Alterações transversais esqueléticas e dentoalveolares (mm/ano) da maxila após terapia com regulador funcional de Frankel II por 12 meses (III': distância entre implantes metálicos posteriores / Mx-Mx': distância entre o ponto mais profundo da concavidade dentoalveolar da maxila do lado direito ao mesmo ponto do lado oposto / A-A': ponto na junção osso alveolar/dente do lado direito as mesmo ponto do lado oposto, conhecido como base apical - BRIEDEN et al., 1984).

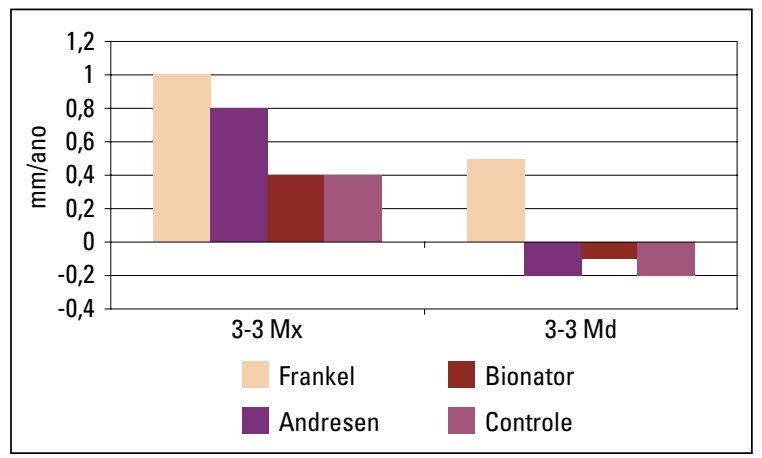

GRÁFICO 2 - Comparação das alterações na distância intercanino da maxila e mandíbula após terapia com diferentes aparelhos funcionais e grupo controle (Média dos trabalhos referidos na tabela 1).

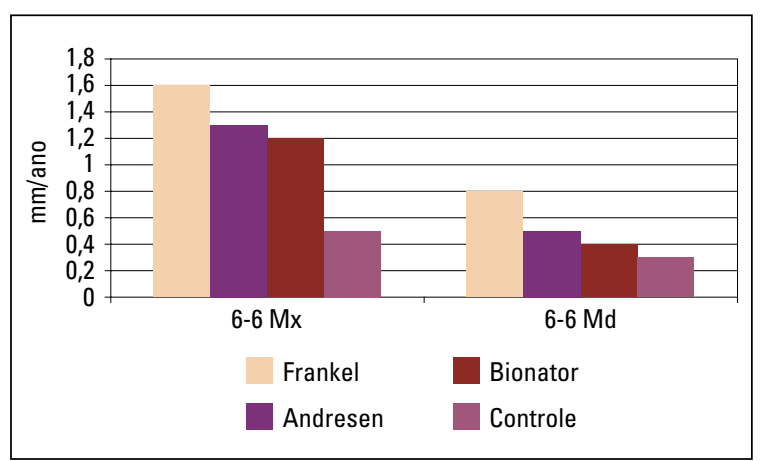

GRÁFICO 3 - Comparação das alterações na distância intermolar da maxila e mandíbula após terapia com diferentes aparelhos funcionais e grupo controle (Média dos trabalhos referidos na tabela 1). 


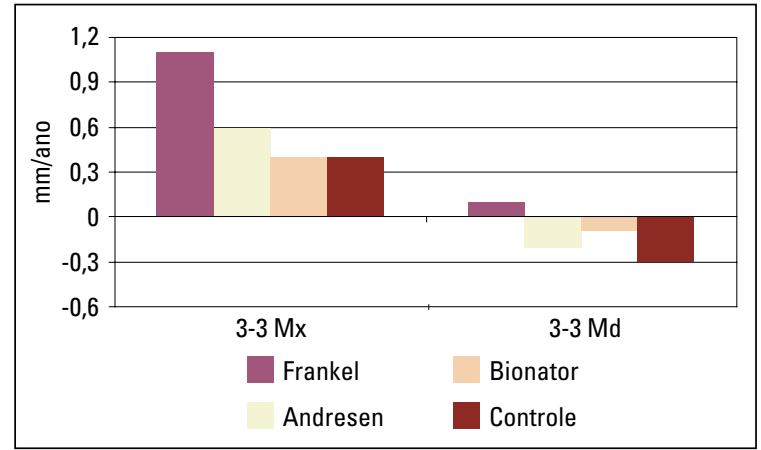

GRÁFICO 4 - Comparação das alterações (mm/ano) na distância intercanino maxilar e mandibular entre diferentes tipos de aparelhos funcionais e amostra controle (GIBBS, HUNT, 1992 e MOYERS, 1976).

processo alveolar normalmente é maior na região maxilar do que mandibular, 3) a densidade do osso maxilar, sendo este mais susceptível às alterações do que o osso mandibular, 4) talvez a pressão oriunda dos músculos da bochecha e lábios atue diferentemente sobre a maxila e mandíbula.

A expansão maxilar é restrita à região dentoalveolar ou também altera a base esquelética? Brieden et al. ${ }^{3}$ avaliaram transversalmente a maxila em diferentes níveis. A maxila recebeu implantes metálicos segundo a técnica descrita por Bjork. A distância entre os implantes e entre pontos pré-estabelecidos na região dentoalveolar e dentária foi mensurada e avaliada após 12 meses de tratamento com o aparelho Frankel (Fig. 4). O grupo experimental apresentou um aumento entre os implantes de $0,57 \mathrm{~mm}$ e na região dentoalveolar de $2,8 \mathrm{~mm}$. A distância entre os implantes para o grupo controle foi menor quando comparada ao grupo experimental, porém não estatisticamente significante. $\mathrm{Na}$ região dentoalveolar para o grupo controle foi observado um aumento de 0,9 mm (Gráf. 1). Nenhum paciente entrou no surto de crescimento pubertário durante o período estudado. A angulação dos primeiros molares superiores praticamente permaneceu inalterada com modificações no máximo de 2 graus. O uso do aparelho Frankel

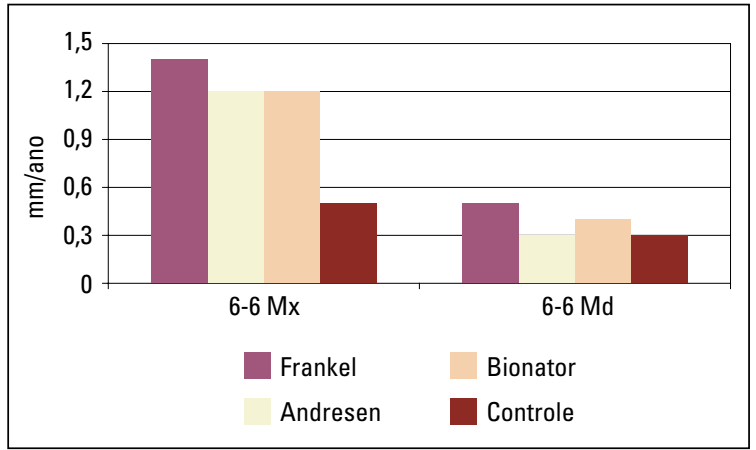

GRÁFICO 5 - Comparação das alterações (mm/ano) na distância intermolar maxilar e mandibular entre diferentes tipos de aparelhos funcionais e amostra controle (GIBBS, HUNT, 1992 e MOYERS, 1976).

provocou um significante aumento na dimensão transversal da maxila principalmente na região apical e região de processo alveolar. Na região apical, o aumento ocorreu principalmente nos últimos 6 meses de tratamento e o aumento atribuído à sutura palatina foi pequeno, porém consistente. Já que as alterações dentárias foram praticamente nulas e os pacientes ainda se encontravam na dentadura mista os autores concluíram que os dentes permanentes iriam erupcionar em arcos com bases mais amplas e sólidas. Outros trabalhos também encontraram uma significante expansão do arco dentário, porém restrita à região posterior e principalmente na maxila ${ }^{1,5}$. Parece sensato afirmar que os aparelhos funcionais são capazes de expandir os arcos dentários, porém não são capazes de realizar uma expansão esquelética na maxila, já que não existe na literatura trabalho com metodologia adequada para esse fato. Mas já foi observado, em estudos ainda em andamento ${ }^{2}$ com implantes metálicos inseridos na região anterior e posterior da maxila, que o aparelho funcional bionator de Balters após 12 meses de tratamento foi capaz de causar uma expansão esquelética estatisticamente significante na região posterior da maxila. Partindo do princípio que o bionator de Balters é um aparelho sólido e de pouca flexibilidade, talvez ainda melhores resultados possam ser encontrados com 
aparelhos funcionais mais flexíveis e ativados para essa finalidade.

É possível uma expansão esquelética mandibular? Novamente a ausência de trabalhos com esse propósito não nos permite concluir uma resposta concisa. Owen ${ }^{21}$, em seus estudos com aparelhos Frankel, observou um aumento da base mandibular. O autor explicou que talvez esse aumento seja resultado do relaxamento do músculo bucinador e dos músculos adjacentes devido ao fato da mandíbula ter ocupado uma nova posição, mais anterior e próxima da normalidade. Infelizmente não podemos afirmar que a terapia funcional causou uma real expansão na base óssea mandibular. Foi comprovado que uma alteração muscular é capaz de provocar uma remodelação óssea mandibular com aposição na região antigoniano e conseqüente expansão dos pontos cefalométricos nessa região, mas para uma real avaliação seria necessária a presença de implantes metálicos inseridos na base da mandíbula, o que infelizmente não encontramos na literatura. Novamente, ainda em estudos em andamento com implantes metálicos inseridos na mandíbula e terapia funcional com o aparelho bionator de Balters, foi observada uma expansão esquelética da mandíbula, porém a diferença não foi estatisticamente diferente do grupo controle. Esses resultados são oriundos de uma amostra pequena e avaliados sobre um curto período. Talvez diferenças significantes possam ser encontradas após maior tempo de tratamento, ou com uma amostra de pacientes maior ou ainda com aparelhos mais flexíveis e ativados para essa finalidade.

É possível alterar o padrão de erupção dos dentes com o uso de aparelhos funcionais? Sim. Baseado na afirmação de que as maiores alterações dentoalveolares acontecem durante o período de erupção dos dentes permanentes, Frankel ${ }^{6,7}$ afirma que é possível alterar o padrão de erupção dos dentes desde que o tratamento coincida com o período de transição dentária. Em um estudo comparativo realizado em 84 pacientes tratados com o aparelho Frankel foi observado um aumento transversal significativo durante a erupção entre as coroas e raízes dos caninos e pré-molares inferiores, como também uma diminuição da inclinação distal dos caninos. Os resultados do estudo suportam os conceitos da matriz funcional de Moss, de que o tamanho e forma da cápsula bucal é um importante fator no desenvolvimento da dentição e que os escudos vestibulares eliminam ou diminuem a pressão da musculatura peribucal permitindo uma alteração do vetor de erupção dos dentes permanentes (Fig. 2).

Qual é a diferença entre os efeitos quando utilizados diferentes aparelhos funcionais? Observe os gráficos 2 e 3 , nele foram anualizados somente trabalhos com os aparelhos Frankel ${ }^{6,11,12}$, 14,15,17,18,21 Andresen ${ }^{12,22,24,27}$ e Bionator ${ }^{12}$ e comparados com grupo controle ${ }^{20}$. De uma maneira geral o aparelho Frankel apresenta os maiores valores, seguido pelos aparelhos Andresen e Bionator. A maior discrepância foi observada na distância intercaninos inferiores com o aparelho Frankel mostrando uma expansão 3 vezes maior que os demais aparelhos, uma possível explicação para essa diferença está no padrão de erupção dos caninos, normalmente alterada quando do uso do aparelho Frankel. Gibbs e Hunt ${ }^{12}$ estudaram os efeitos de diferentes aparelhos funcionais sobre a largura dos arcos dentários. Realizado na forma retrospectiva, os autores selecionaram somente pacientes com má oclusão Classe II, divisão 1, e formaram 3 diferentes grupos de acordo com o aparelho empregado. Vinte e sete pacientes foram tratados com aparelho funcional Andresen, 19 pacientes tratados com o aparelho Bionator e 23 pacientes tratados com o aparelho Frankel. Modelos de estudo foram avaliados no início do tratamento, no final do tratamento e 1 ano de pós-tratamento com contenção. A média de idade dos pacientes no início do tratamento variava entre 11,7 a 12,2 anos de idade. O estudo mostrou que os três aparelhos produziram expansão maxilar e que essa expansão foi maior para a 
região dos molares do que para a região dos caninos. Os maiores valores foram observados no grupo do aparelho Frankel. A média da distância intermolares superiores foi de $1,2 \mathrm{~mm}, 1,2 \mathrm{~mm}$ e 1,4 $\mathrm{mm}$ e a distância intercaninos foi de 0,6 $\mathrm{mm}, 0,4 \mathrm{~mm}$ e $1,1 \mathrm{~mm}$ respectivamente para os grupos Andresen, Bionator e Frankel (Gráf. 4, 5). A recidiva da distância intercaninos foi igual para os três grupos na mandíbula e maior na maxila para o grupo Frankel. $\mathrm{Na}$ mandíbula, a distância intermolares aumentou em média $0,3 \mathrm{~mm}, 0,4$ $\mathrm{mm}$ e $0,5 \mathrm{~mm}$ para os grupos com aparelhos Andresen, Bionator e Frankel. De uma maneira geral os pacientes do grupo Frankel apresentaram metade da recidiva observada nos outros dois grupos. Embora o apinhamento possa ser amenizado durante o tratamento, os resultados mostraram que particularmente no arco inferior, mínimo ou nenhum ganho pode ser obtido com o tratamento funcional em pacientes nessa fase de maturação óssea. De uma maneira geral o aparelho Frankel pareceu ser mais eficiente quando considerado as alterações transversais dos arcos dentários. Acreditamos que talvez alguns fatores presentes nesse aparelho o fizeram superior aos outros, como por exemplo, a flexibilidade do aparelho e/ou o tamanho dos escudos vestibulares.

Qual e a melhor época para o tratamento? A resposta para essa pergunta está diretamente relacionada com os objetivos do tratamento. Por exemplo, caso um dos objetivos seja alterar o padrão de erupção e o desenvolvimento do arco dentário, o tratamento deverá ocorrer durante o período de transição dentária, ou seja, durante a dentadura mista; caso queira potencializar os efeitos esqueléticos o tratamento deverá coincidir com os períodos de maior crescimento mandibular durante os surtos juvenil ou pubertário, ou ainda caso as alterações almejadas sejam de caráter dentoalveolar, o tratamento não depende necessariamente coincidir com os surtos de crescimento. Alguns autores preconizam o tratamento durante o final da dentadura mista após
10 anos de idade, levando em consideração o período de erupção dos dentes permanentes, crescimento mandibular e o fato de que ao término dessa fase de tratamento o paciente não precisaria passar por um período de contenção antes de iniciar a segunda fase com aparelho fixo. A segunda fase começaria imediatamente ao término da primeira, servindo como período de contenção para a primeira, além de diminuir o tempo total de tratamento ${ }^{5,11}$. Evidente que os casos devem ser avaliados individualmente e tratados no período de maior conveniência.

\section{CONCLUSÃO}

A literatura nos permitiu concluir que é possível alterar a dimensão transversal da maxila e mandíbula com o uso de aparelhos ortopédicos funcionais em pacientes com crescimento. As alterações transversais observadas ocorrem simultaneamente às alterações ântero-posteriores e verticais. Não nos parece sensato utilizar o aparelho ortopédico funcional com a exclusiva função de corrigir uma discrepância transversal. Clinicamente, os melhores resultados foram obtidos na expansão da distância intermolares, principalmente na maxila. As alterações apresentadas foram dentoalveolares apesar de poucos trabalhos investigarem as reais alterações esqueléticas. Recentes estudos comprovam que é possível uma expansão esquelética da maxila decorrente de uma terapia funcional. Com certeza isso não seria uma solução imediata para a falta de espaço nos arcos dentários, mas seguramente seria um novo campo a ser explorado.

\section{AGRADECIMENTO}

À CAPES (Bolsista Doutorado-Sanduíche) e à professora Dra. Ana Claudia Moreira Melo pela revisão do trabalho. 


\title{
The effects of functional appliances in the maxilla and mandibular transverse dimension
}

\begin{abstract}
While the effects of functional appliances on the antero-posterior and vertical dimensions have been well studied, the literature pertaining to their transverse effects is limited and has not previously been synthesized. Buccal muscular pressure, periostal traction, and tongue repositioning are some of the proposed mechanisms by which transverse jaw dimensions could change during treatment. Various studies have reported transverse dental arch changes, but the effects appear to be dependent on the functional appliance used. Based on a systematic review of the literature, the purpouse of this paper are: 1) evaluated the proposed mechanisms that influence the transverse dimensions during treatment and 2) summarizes the clinical effects of various functional appliances on transverse jaw dimensions.
\end{abstract}

Key words: Functional appliance. Maxillary expansion. Mandibular expansion. Class II malocclusion.

\section{REFERÊNCIAS}

1. ALMEIDA, M. A. A. FERREIRA V. M. G. FALTIN JÚNIOR, K. Expansão espontânea dos arcos dentários no tratamento da Classe II mandibular com Bionator de Balters. Ortodontia Gaúcha, Porto Alegre, v. 2, p. 4-15, 1998.

2. ARAUJO, A. M. Avaliação das alterações esqueléticas transversais da maxila e da mandíbula no tratamento da má oclusão Classe II divisão 1 com bionator de Balters: estudo cefalométrico com implantes metálicos.2003. Dissertação (Doutorado)-Faculdade de Odontologia, UNESP, Araraquara, 2003.

3. BRIEDEN, C. M.; PANGRAZIO-KULBERSH, V.; KULBERSH, R. Maxillary skeletal and dental change with Frankel appliances: an implant study. Angle Orthod, Appleton, v. 54, p. 226-232, 1984

4. CHRISTIANSEN, R. L.; EVENS, C. A.; SUE, S. K. Resting pressure tongues. Angle Orthod, Appleton, v. 49, p. 92-97, 1979.

5. DOMINGUEZ, G. C.; HIRSCHHEIMER, C.; VIGORITO, J. W. Alterações transversais das arcadas dentárias decorrentes do tratamento com bionator em pacientes em crescimento com maloclusão Classe Il divisão primeira. Ortodontia, São Paulo, v. 34, p. 36-42, 2001.

6. FRANKEL, R. Decrowding during eruption under the screening influence of vestibular shields. Am J Orthod, St. Louis, v. 65, p. 372-405, 1974

7. FRANKEL, R. The guidance of eruption without extraction. Trans Eur Orthod Soc, London, p. 303-313, 1971.

8. FRANKEL, R.; MULLER, M.; FALCK, F. The uprighting effect of the Frankel appliance on the mandibular canines and premolars during eruption. Am J Othod Dentofacial Orthop, St. Louis, v. 92, p.109116, 1987.

9. GHAFARI, J. Palatal sutural response to buccal muscular displacement in the rat. Am J Othod Dentofacial Orthop, St. Louis, v. 85, p. 351-356, 1984

10. GHAFARI, J.; SHANFELD. J. Effect of removing buccal musculature pressure from the rat maxilla. J Dent Res, v. 60, p. 432, 1981

11. GHAFARI, J.; SHOFER, F. S.; JACOBSSON-HUNT, U.; MARKOWITZ, D. L.; LASTER L. L. Headgear versus function regulator in the early treatment of Class II, division 1 malocclusion: A randomized clinical trial. Am J Othod Dentofacial Orthop, St. Louis, v. 113, p. 51-61, 1998.

12. GIBBS, S. L.; HUNT, N. P. Functional appliances and arch width. $\mathbf{B r} \mathbf{J}$ Orthod, v. 19, p.117-125, 1992.

13. GRABER, T. M.; VANARSDALL JR., R. L. Orthodontics: current principles and techniques. 2nd ed. St. Louis: C. V. Mosby, 1994. p. 965.

14. HAMILTON, S. D.; SINCLAIR, P. M.; HAMILTON, R. H. A cephalometric, tomographic and dental cast evaluation of the Fränkel therapy. Am J Othod Dentofacial Orthop, St. Louis, v. 92, p. 427-434, 1987.
15. HIME, D. L. OWEN, A. H. The stability of the arch expansion effects of Frankel appliance therapy. Am J Othod Dentofacial Orthop St. Louis, v. 98, p. 437-445, 1990.

16. KALOGIROU, K.; AHLGREN, J.; KINGE, B. Effects of the buccal shields on the maxillary dentoalveolar structures and the midpalatal suture: histologic and biometric studies in rabbits. Am J Othod Dentofacial Orthop, St. Louis, v. 109, p. 521-530, 1996.

17. McDOUGALL, P. D.; McNAMARA J. A.; DIERKES. J. M. Arch width development in class II patients treated with the Frankel appliance. Am J Othod, St. Louis, v. 82, p.10-22, 1982

18. McWADE, R. A.; MAMANDRAS, A. H.; HUNTER, W. S. The effects of Frankel II treatment on arch width and arch perimeter. Am J Othod Dentofacial Orthop, St. Louis, v. 92, p. 313-320, 1987

19. McWADE, R. A.; MAMANDRAS, A. H.; HUNTER, W. S. The effects of Frankel II treatment on arch width and arch perimeter. Am J Orthod Dentofacial Orthop, St. Louis, v. 92, no. 4, p. 313-320, Oct.1987.

20. MOYERS, R.; VAN DER LINDEN, F.; RIOLO, M. Standards of human occlusal development. In: CARLSON, D. S. Cranio Facial Biology. Michigan: [s. n.], 1976. p. 371.

21. OWEN, A. H. Morphologic changes in the transverse dimension using the Frankel appliance. Am J Othod Dentofacial Orthop St. Louis, v. 83, p. 200-216, 1983

22. PANCHERZ, H. Long-term effects of activator: Andresen appliance treatment. Odontol Revy, Lund, v. 27, no. 35, 35, 1976. Spplement.

23. PROFFIT, W. R. Muscle pressure and tooth position. North América whites and Australian aborígines. Angle Orhtod, Appleton, v. 45 p.1-11, 1975

24. REEY, R. W.; EASTWOOD, A. The passive activator: case selection, treatment response and corrective mechanics. Am J Othod, St. Louis, v. 73, p. $378-409,1978$

25. SOTIRIADOU, A. D.; JOHNSTON, L. E. Expansion with vestibular shields: an experiment test of the periostal-pull hypothesis. Semin Orthod, Philadelphia, v. 5, p.121-127, 1999

26. TRENOUTH, M. J. A functional appliances system for the correction of Class II relationship. Br J Dent, London, v. 16. p. 169-176, 1989.

27. VARGERVIK, K. Morphologic evidence of muscle influence on dental arch width. Am J Othod, St. Louis, v. 76, p. 21-28, 1979.

\section{Endereço para correspondência}

Adriano Marotta Araujo

Rua Marcondes Salgado, 64

São José dos Campos - SP

CEP: 12243-820

E-mail: dradrianoaraujo@hotmail.com 